\title{
Wound infection secondary to snakebite
}

\author{
M Wagener, ${ }^{1,2} \mathrm{MB} \mathrm{ChB} ; \mathrm{M}$ Naidoo, ${ }^{2} \mathrm{MB} \mathrm{ChB}, \mathrm{FCS}(\mathrm{SA}) ;$ C Aldous, ${ }^{2} \mathrm{PhD}$ \\ ${ }^{1}$ Department of Paediatric Surgery, School of Clinical Medicine, College of Health Sciences, Nelson R Mandela School of Medicine, \\ University of KwaZulu-Natal, Durban, South Africa \\ ${ }^{2}$ College of Health Sciences, Nelson R Mandela School of Medicine, University of KwaZulu-Natal, Durban, South Africa
}

Corresponding author: M Wagener (mark.wagener2@gmail.com)

\begin{abstract}
Background. Snakebites can produce severe local and systemic septic complications as well as being associated with significant overall morbidity and even mortality.

Objective. A prospective audit was undertaken to determine the bacterial causation of wound infection secondary to snakebite, and attempt to quantify the burden of disease.

Methods. The audit was undertaken at Ngwelezane Hospital, which provides both regional and tertiary services for north-eastern KwaZuluNatal Province, South Africa, over a 4-month period. Records of patients who required surgical debridement for extensive skin and softtissue necrosis were analysed. At the time of debridement, tissue samples of necrotic or infected tissue were sent for bacteriological analysis as standard of care. Microbiology results were analysed.

Results. A total of 164 patients were admitted to hospital for management of snakebite, of whom 57 required surgical debridement and 42 were included in the final microbiological analysis. Children were found to be the most frequent victims of snakebite; $57.8 \%$ of patients in this study were aged $\leq 10$ years and $73.7 \% \leq 15$ years. Culture showed a single organism in $32 / 42$ cases, two organisms in 8 and no growth in 2. Eight different types of organisms were cultured, five of them more than once. Thirty-five specimens (83.3\%) grew Gram-negative Enterobacteriaceae, the most frequent being Morganella morganii and Proteus species. Thirteen specimens (31.0\%) grew Enterococcus faecalis. Gram-negative Enterobacteriaceae showed $31.4 \%$ sensitivity to ampicillin, $40.0 \%$ sensitivity to amoxicillin plus clavulanic acid, $34.3 \%$ sensitivity to cefuroxime, $97.1 \%$ sensitivity to ceftriaxone, and $100 \%$ sensitivity to ciprofloxacin, gentamicin and amikacin. E. faecalis was $92.3 \%$ sensitive to amoxicillin, $92.3 \%$ sensitive to amoxicillin plus clavulanic acid, $100 \%$ sensitive to ciprofloxacin, $92.3 \%$ resistant to erythromycin and $100 \%$ resistant to ceftriaxone.

Conclusion. Children are particularly vulnerable to snakebite, and the consequences can be devastating. While the majority of patients in this study were shown to have secondary bacterial infection, debridement and subsequent wound management is considered the mainstay of treatment. Common organisms are Enterobacteriaceae and enterococci. There appears to be a role for antibiotics in the management of these patients. A good antibiotic policy is strongly advocated.
\end{abstract}

S Afr Med J 2017;107(4):315-319. DOI:10.7196/SAMJ.2017.v107i4.12084

Snakebites can produce severe local and systemic septic complications in addition to the expected morbidity and even mortality caused by venom alone. In the majority of snakebite victims presenting to hospital, envenomation involves painful progressive swelling as the predominant symptom. ${ }^{[1,2]}$ Other envenomation syndromes, including acute life-threatening coagulopathy, muscle weakness and respiratory paralysis, are rare. ${ }^{[1-4]}$ Cases of severe envenomation are often characterised by subsequent wound infection and skin and softtissue necrosis. ${ }^{[1,2]}$

While the role of antivenom administration in the treatment of acute envenomation has been well described, ${ }^{[4]}$ treatment of the complex wounds that result from subsequent necrosis and infection needs further investigation. In a 1999 study by Blaylock ${ }^{[5]}$ at Eshowe District Hospital, KwaZulu-Natal (KZN) Province, South Africa (SA), the bacterial aetiology of wound infection secondary to snakebite was described. Gram-negative aerobic Enterobacteriaceae were the most commonly isolated organisms in that study, but antibiotic susceptibility was not reported. In areas of high snakebite prevalence outside SA, a similar spectrum of bacterial species has been found and antibiotic sensitivities reported. ${ }^{[6-9]}$ The bacterial cause of infected snakebite wounds in northern KZN has been determined by previous work, but antibiotic susceptibility remains untested. A current description of associated morbidity is also required.

Treatment of complex wounds resulting from cytotoxic snake envenomation poses multiple challenges. A multidisciplinary approach is required. Current treatment strategies need to be examined in order to reduce morbidity.
Numerous authors have recognised that the public health impact of snakebite has been underestimated. ${ }^{[9-12]}$ Importantly, since the type of snake responsible for the majority of severe envenomation will vary between regions, the clinical problems associated with snakebite will also be unique for each region. The main envenomation syndrome in KZN is cytotoxicity. ${ }^{[1,2]}$ It is postulated that the tissue destruction, disfigurement, amputation and physical disability that result from predominantly cytotoxic snake envenomation are responsible for the majority of the untold burden of disease in our region. ${ }^{[13,14]}$ Indices to quantify this morbidity include length of hospital stay and objective assessment of resulting physical disability. ${ }^{[15]}$

The snakes that cause the greatest human suffering are those that bite frequently and have bites resulting in severe envenomation. ${ }^{[9,14]}$ Snakes that bite frequently are those with behaviour associated with a high incidence of human contact. Snake species with these characteristics vary between regions. ${ }^{[9]}$ The types of snake responsible for the majority of morbidity in our study population were examined.

Rural populations are at the greatest risk of snakebite. ${ }^{[10-12]}$ Of particular concern is that in rural communities, the majority of snakebite victims are children. ${ }^{[1-4]}$ The reasons for this phenomenon need to be examined. Novel strategies used to prevent bites may be possible, thus reducing long-term social and economic consequences.

\section{Objective}

A prospective audit was undertaken to determine the bacterial causation of wound infection secondary to snakebite in KZN, as well as attempt to quantify the burden of disease. 


\section{Methods}

The study was conducted at Ngwelezane Tertiary Hospital, situated in a semi-rural settlement outside the town of Empangeni, $\mathrm{KZN}$, and serving a predominantly rural population. It is the referral centre for 22 peripheral hospitals and sees an alarming number of snakebites each year, ${ }^{[1,2]}$ mainly in the summer months. ${ }^{[1-4]}$

During the 4-month period December 2014 - March 2015, patients requiring surgical debridement of snakebite were enrolled prospectively. No new protocols were put in place for the purpose of the study, and study information represented standard patient management. A sample of necrotic tissue was taken from each patient and sent for bacteriological analysis. Pathogens isolated and antibiotic susceptibilities were recorded. In vitro culture of debrided tissue was done using blood agar and MacConkey agar culture media, and susceptibility testing was performed using the disc-diffusion method as per standard National Health Laboratory Service protocols.

Other data obtained to determine morbidity included anatomical location of debridement, extent of debridement, need for re-debridement, method of wound closure, skin graft take, length of hospital stay and objective functional impairment. Each patient, or the caregiver if the victim was a child, was questioned on the type of snake they had been bitten by. Age and sex of victims were recorded.

Patients were included in the analysis of overall treatment but excluded from microbiological analysis if the wound had broken down spontaneously or had been informally debrided elsewhere, specimens had been lost or incorrectly processed, or the debridement had been done for skin necrosis only without any evidence of infection.

Data from each patient were recorded on an individual data collection pro forma. Data were then inserted into an Excel spreadsheet, version 15.27 (Microsoft, USA), for analysis.

Ethics approval was obtained from the Biomedical Research Ethics Committee of the University of Kwa-Zulu Natal. (ref. no. BE006/15).

\section{Results}

\section{Description of the sample}

A total of 164 patients were admitted with snakebite wounds during the study period, 57 (34.8\%) of whom required surgical debridement. Fifteen patients were not included in the final microbiological analysis for the following reasons: (i) the wound had broken down spontaneously or been informally debrided elsewhere
( $n=5$ patients); (ii) tissue specimens had been lost $(n=6)$; (iii) tissue specimens had been incorrectly processed $(n=2)$; and (iv) debridement had been done for skin necrosis without any evidence of infection, and microbiological analysis confirmed no bacterial growth $(n=2)$.

Thirty-three patients (57.9\%) were referred from rural district hospitals, either acutely $(n=19,57.6 \%)$ or as non-urgent cases referred to the department of surgery with wound complications after spending time at a peripheral hospital ( $n=14,42.4 \%)$. Those with delayed referral spent a mean of 8.85 days (range $3-20$ ) at the peripheral hospital prior to referral. caregivers claimed to have identified the snake with confidence. Of the 28 positively identified snakes, 26 (92.9\%) were Mozambique spitting cobras (Naja mossambica), or ' $M$ 'fezi'.

The most frequent victims of snakebite were found to be children, particularly those
In 28 cases $(49.1 \%)$, patients or their

aged $\leq 10$ years. Most of the victims in this study were aged $\leq 10$ years $(57.8 \%)$, and $73.7 \%$ were aged $\leq 15$ years (Fig. 1 ).

The majority of the bites occurred at night, 33/55 (60.0\%) between $22 \mathrm{~h} 00$ and $08 \mathrm{~h} 00$ and $17 / 55(30.8 \%)$ in the evening between $18 \mathrm{~h} 00$ and $22 \mathrm{~h} 00$. Five bites $(9.1 \%)$ occurred in the daylight hours of $08 \mathrm{~h} 00$ $18 \mathrm{~h} 00$. Most of the victims $(86.0 \%)$ were bitten while sleeping.

\section{Microbiological analysis}

Of the 42 cases included in the final microbiological analysis, $76.2 \%(n=32)$ showed single growth, $19.0 \%(n=8)$ grew two organisms, and in two cases (4.8\%) there was no growth. Eight different types of organisms were cultured, five of them more than once.

Fig. 2 shows the various bacteria cultured. Thirty-five specimens (83.3\%) grew Gramnegative Enterobacteriaceae. Enterococcus faecalis was found in 13 specimens (31.0\%). The two most frequent Gram-negative Enterobacteriaceae were Morganella morg-

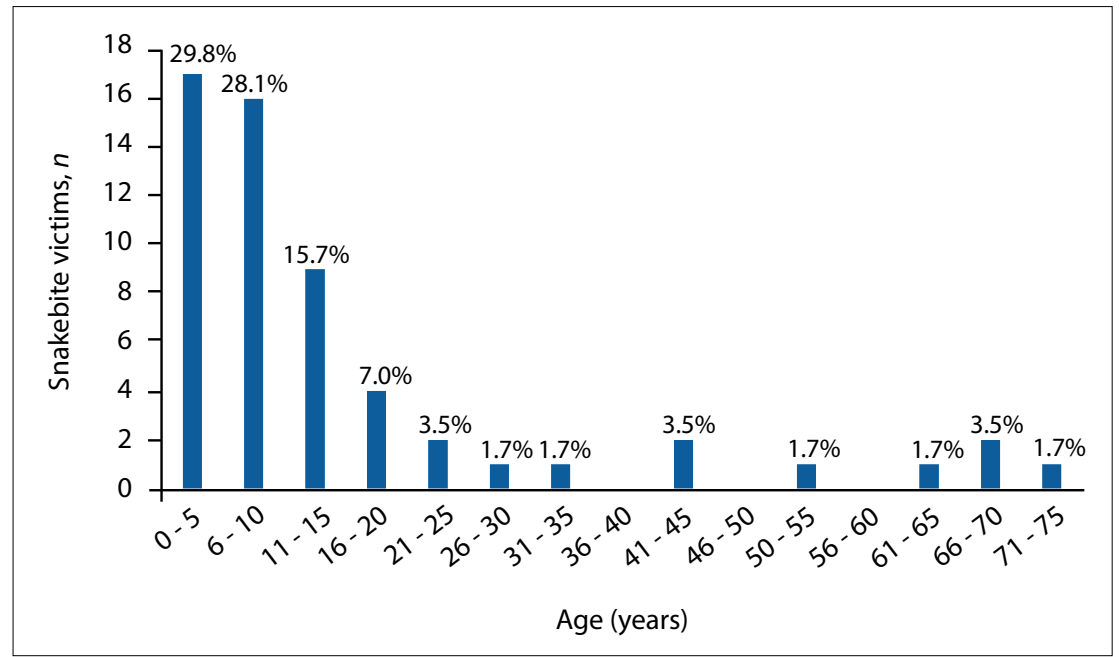

Fig. 1. Age distribution of patients.

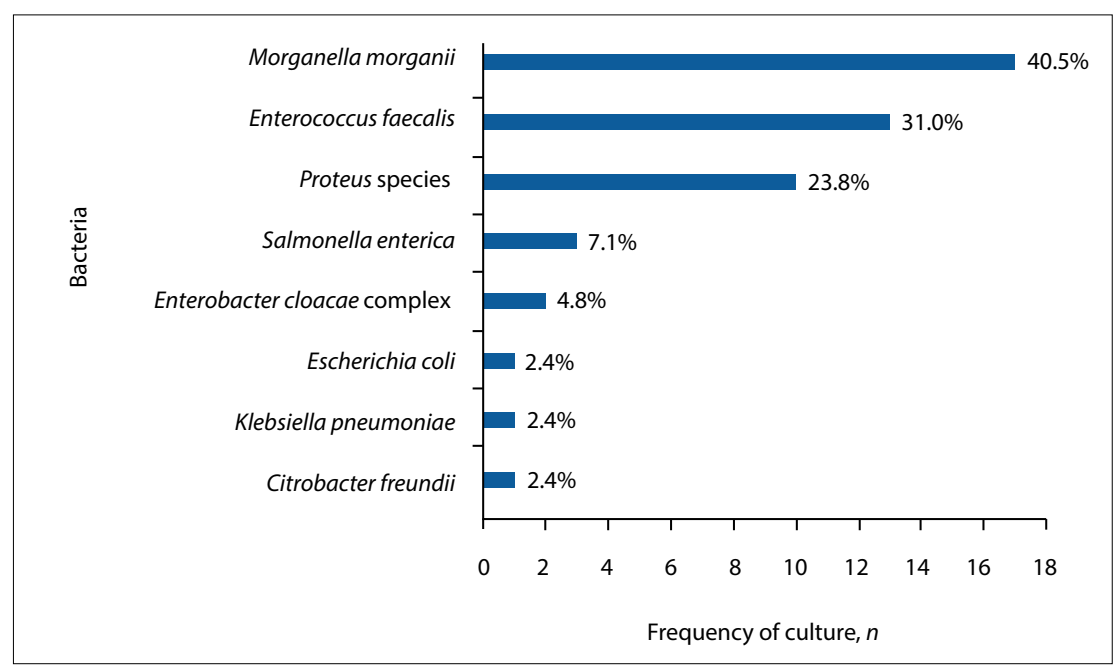

Fig. 2. Organisms grown from infected snakebite wounds. 
anii, which occurred in $40.5 \%$ of specimens, followed by Proteus species, which occurred in $23.8 \%$. No anaerobes were found.

\section{Antibiotic resistance}

Gram-negative Enterobacteriaceae showed $68.6 \%$ resistance to ampicillin, $60.0 \%$ resistance to amoxicillin plus clavulanic acid, and $65.7 \%$ resistance to second-generation cephalosporins. These organisms were $97.1 \%$ sensitive to ceftriaxone and $100 \%$ sensitive to ciprofloxacin and aminoglyosides. E. faecalis showed $92.3 \%$ sensitivity to both ampicillin and amoxicillin plus clavulanic acid, as well as $100 \%$ sensitivity to ciprofloxacin. Enterococci were $92 \%$ resistant to erythromycin and $100 \%$ resistant to cephalosporins.

Thirty-two patients $(56.1 \%)$ received empirical antibiotic therapy, either at presentation or shortly before debridement. Amoxicillin plus clavulanic acid was the most commonly used antibiotic. Cloxacillin, metronidazole, amoxicillin, ceftriaxone and gentamicin were also used (Fig. 3).

\section{Morbidity and treatment outcomes}

Of the 57 patients, $32(56.1 \%)$ sustained upper limb bites, 20 (35.1\%) lower limb bites, 5 (8.8\%) bites to the torso and $2(3.5 \%)$ facial bites, while 1 patient $(1.8 \%)$ was bitten on the occiput of the head. Four patients (7.0\%) sustained multiple bites.

The average delay from injury to surgical debridement was 7.8 days (range 3 -22). Among patients presenting to the hospital within 48 hours of the bite, the delay in debridement was 5.2 days from admission. Sixteen patients $(28.1 \%)$ required re-debridement. In 27 cases (47.4\%) a skin graft was necessary, in 26 cases $(45.6 \%)$ wounds were small enough to heal by secondary intention, and in 4 cases this information was not recorded. Skin graft take was $80-100 \%$ in $20 / 27$ patients $(74.1 \%)$, and only one patient had complete skin graft failure. One patient in this cohort required fasciotomy. Four patients required digital amputations, but there were no major limb amputations. Two patients had severe and life-threatening sepsis as a result of wound infection.

The average length of hospital stay was 31.3 days (range $3-74$ ). Seven patients were assessed as having objective functional impairment in the form of digital amputation $(n=4)$, difficulty in walking $(n=2)$ and impaired elbow flexion $(n=1)$.

\section{Discussion}

The majority of patients in this study were shown to have secondary bacterial infection. The clinical significance of these infections was variable. Clinically significant wound infection was considered to be present in all patients included in the final microbiological analysis. These infections may affect wound healing, skin graft take and possibly even long-term morbidity. While debridement and subsequent wound management is considered the mainstay of treatment, antibiotic therapy is considered to be an important adjunct in these cases. Presence of a systemic inflammatory response as a result of wound infection was not included in the data collection. Two patients had severe and life-threatening sepsis as a result of wound infection.

Gram-negative Enterobacteriaceae and E. faecalis were the most commonly isolated organisms. Gram-negative Enterobacteriaceae showed high resistance to ampicillin (68.6\%), amoxicillin plus clavulanic acid (60.0\%), and second-generation cephalosporins (65.7\%). Sensitivity was adequate to ceftriaxone (97.1\%) and ciprofloxacin, gentamicin and amikacin (all 100\%). E. faecalis showed good sensitivity to

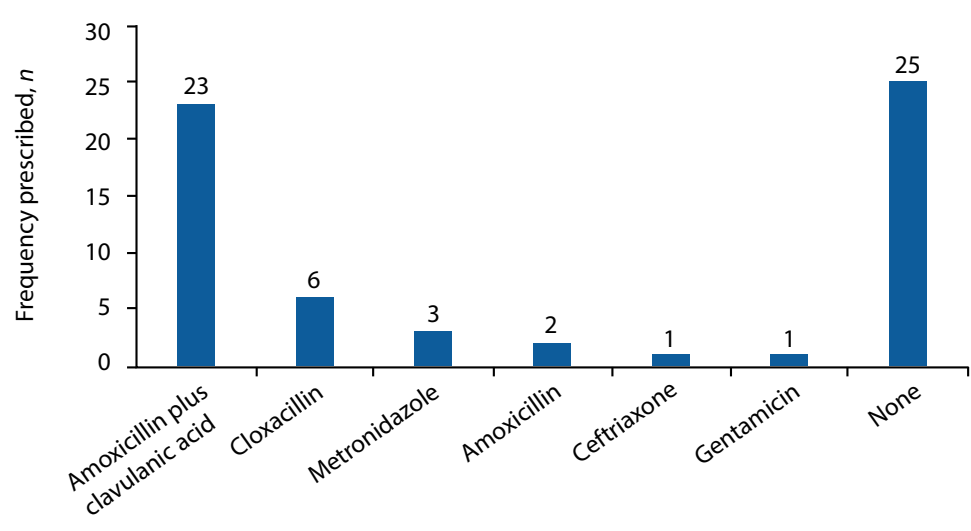

Antibiotic ampicillin (92.3\%), amoxicillin plus clavulanic acid (92.3\%) and ciprofloxacin (100\%).

We believe that cultured organisms represent true infection rather than contaminants, because the organisms grown are similar to those found in mouths of KZN snakes ${ }^{[16]}$ and because wounds that had either broken down or been informally debrided elsewhere were excluded from the microbiological analysis.

Current recommendations advise against the prophylactic use of antibiotics in snakebites. ${ }^{[4,17]}$ These recommendations are based on research showing a paucity of bacteria in snakes' mouths and that venom has antibacterial properties. ${ }^{[16,18]}$ There does, however, appear to be a role for antibiotics in the managenent of severe local and systemic septic complications from snakebite, and we conclude that antibiotics play an important role in therapy of infected snakebite wounds.

This study demonstrated that use of antibiotics in the management of snakebites was widespread and not directed. Amoxicillin plus clavulanic acid was the most commonly used antibiotic, to which Gramnegative bacteria from wounds cultured in this series showed $60.0 \%$ resistance. Cloxacillin was the next most commonly used antibiotic, and would have been of no benefit because staphylococcal infection has not been shown to occur in these patients. In cases where empirical therapy is deemed necessary, antibiotics need to cover Gram-negative Enterobacteriaceae and E. faecalis. According to these data, Gram-negatives would be covered by ciprofloxacin, an aminoglycoside or a thirdgeneration cephalosporin. E. faecalis would be adequately covered by ampicillin or ciprofloxacin. A good antibiotic policy is strongly advocated.

Snakebite wounds are particularly unforgiving wounds to treat. There is often exposure of non-granulating surfaces and superimposed infection. In addition, victims are frequently young children who comply poorly with rehabilitation and pose challenges for dressing changes (Fig. 4). Upper limb and torso injuries pose particular difficulties. Nevertheless, by applying standard wound care principles, involving rehabilitation professionals early on and using a combination of delayed primary closure and skin grafting, it is possible to achieve a satisfactory result.

This study demonstrates the significant burden of disease caused by cytotoxic snakebite. This can be quantified firstly by length of hospital stay, (average 31.3 days). 


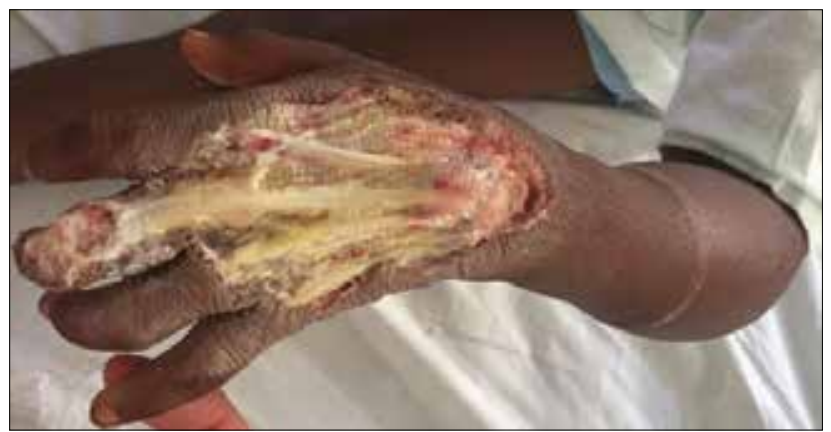

Fig. 4. Exposure of tendons in a necrotic septic snakebite wound after debridement.

Additionally, patients in this study showed objective functional impairment in terms of digital amputation ( $n=4$ cases), difficulty in walking $(n=2)$ and impaired upper limb mobility $(n=1)$. Of concern is that many patients, especially children, suffer permanent functional impairment that could not be adequately quantified by methods used in this study. Children with devastating extremity injuries are likely to have difficulty learning basic life skills, playing and performing at school.

With focused care and more aggressive treatment and rehabilitation, we believe that the average length of hospital stay can be reduced. When patient care is evaluated using the results of this study, this will be an area to focus on.

Victims' identification of the offending snake has been described as unreliable in the literature, ${ }^{[2,4]}$ but in this study $49.1 \%$ of patients or their caregivers confidently identified the snake, the majority being identified as M'fezi. Our opinion is that the majority of these reports are accurate. Most bites ascribed to the M'fezi occurred at night while sleeping, which is in keeping with the behaviour of this snake. ${ }^{[9,19,20]}$ Secondly, many wounds showed characteristic features of M'fezi bites: necrosis involving skin and subcutaneous soft tissue, with patches of skin necrosis separated by normal-looking skin producing characteristic 'skip lesions' (Fig. 5) ${ }^{[9,19,21]}$ or with extensive undermining of tissues (Fig. 6). Lastly, the snake is justifiably feared by the local population and is therefore well known.

This study demonstrates that rural populations are most at risk of snakebite: $57.9 \%$ of victims were referred from outlying rural hospitals. The likelihood of humans coming into contact with snakes is considerably increased in rural areas. Rural-dwelling families are at risk in their homes, and farmers and herders are at risk in their occupation.

Confirming what has been found in previous audits, children are particularly vulnerable to snakebite. ${ }^{[1-4]}$ This has traditionally been thought to be a consequence of children's naturally inquisitive behaviour, ${ }^{[2,4]}$ but since the majority of patients in this study were bitten at night while sleeping, another explanation is needed. It is possible that these bites occur because children in our rural communities often sleep on the floor, while more senior members of the household occupy a bed. In addition to children being particularly vulnerable to snakebite, local and systemic envenomation syndromes occur more frequently and with greater severity in this age group. ${ }^{[4]}$ This is probably due to the higher venom-to-mass ratio, as suggested by Blaylock. ${ }^{[1,16]}$

The numbers in this study were higher than those collected by Wood et al. ${ }^{[4]}$ in their series from the same hospital in 2007/2008. That study recorded the highest number of snakebites in the months December - March, these being the only months during which data were collected in our study. During the same time

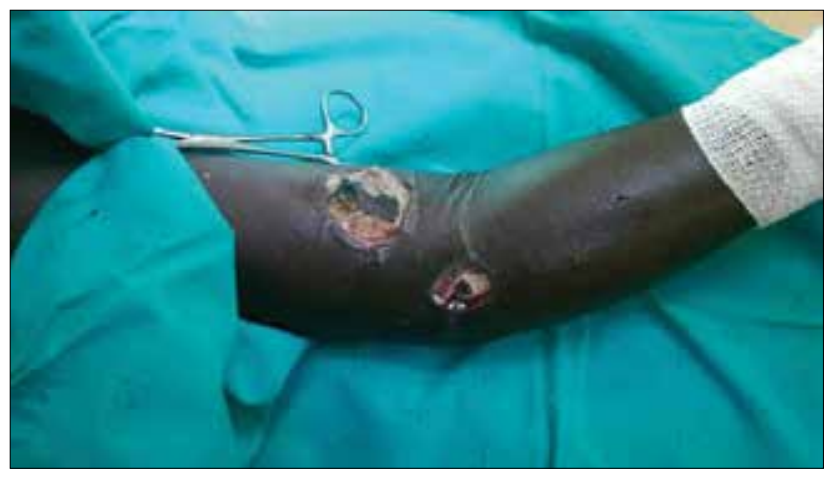

Fig. 5. Characteristic 'skip lesions' of M'fezi bite.

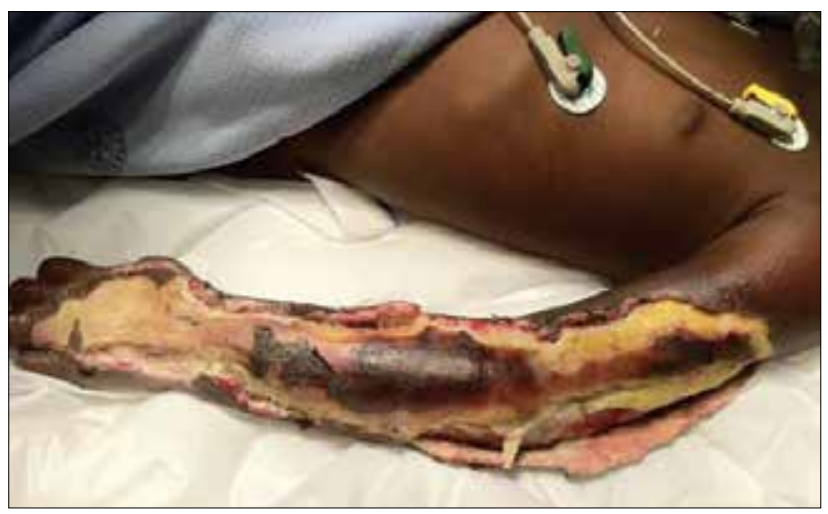

Fig. 6. Necrotic snakebite wound presumed to be from M'fezi bite.

frame, Wood et al. had 132 patients, compared with 164 in our study. The reason for the discrepancy is likely to be twofold. Firstly, in 2007/2008 Wood et al. examined only patients presenting to the emergency department of Ngwelezane Hospital, while a number of patients in our study were referred to the department of surgery as non-urgent cases with wound complications after spending time at a peripheral hospital. Secondly, since 2012 referral patterns have changed, resulting in a greater number of district hospitals referring to Ngwelezane. There are no previous studies from Ngwelezane examining the number of snakebite victims requiring surgical debridement.

A delay of 5 - 7 days before debridement of necrotic snakebite wounds is recommended to allow demarcation of dead tissue. ${ }^{[16,22]}$ In this study, this recommended time frame was achieved in patients presenting acutely to our hospital, with an average of 5.2 days to debridement. However, patients referred from elsewhere experienced a longer delay to debridement owing to time spent at a peripheral hospital and time required for treatment with antibiotics after arriving at our hospital (Fig. 7), accounting for the longer overall delay to debridement for patients in this study. Two patients in our study experienced cardiorespiratory collapse as a complication of grossly infected wounds (Fig. 8), and in these cases we performed the debridement as an emergency in order to achieve source control in the treatment of severe sepsis.

The role of antivenom in preventing these devastating local and regional complications of snakebite is largely unknown. In a randomised, double-blind, placebo-controlled trial, antivenom was shown to accelerate oedema resolution in bites from green pit vipers in Thailand. ${ }^{[23]}$ Antivenom has also been demonstrated to decrease compartment pressure with rattlesnake envenomation in an animal model from the USA. ${ }^{[24]}$ However, these effects remain untested in Africa. The efficacy of antivenom in reducing skin and 


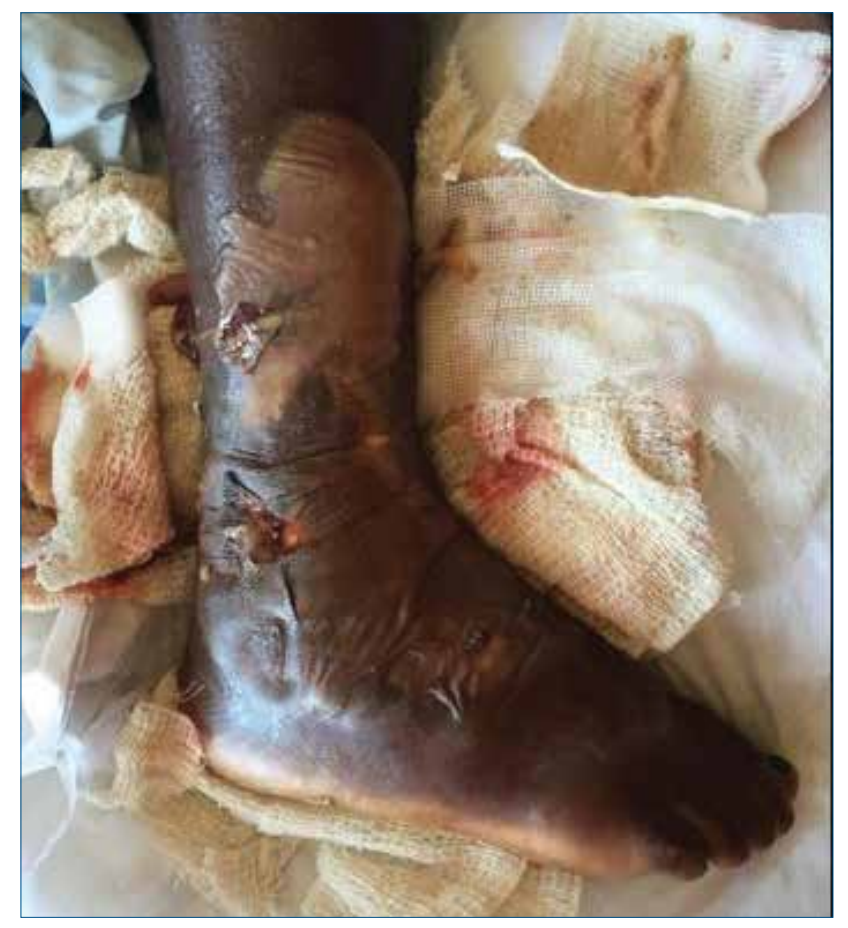

Fig. 7. Patient referred with septic snakebite wound after being managed expectantly at a peripheral hospital.

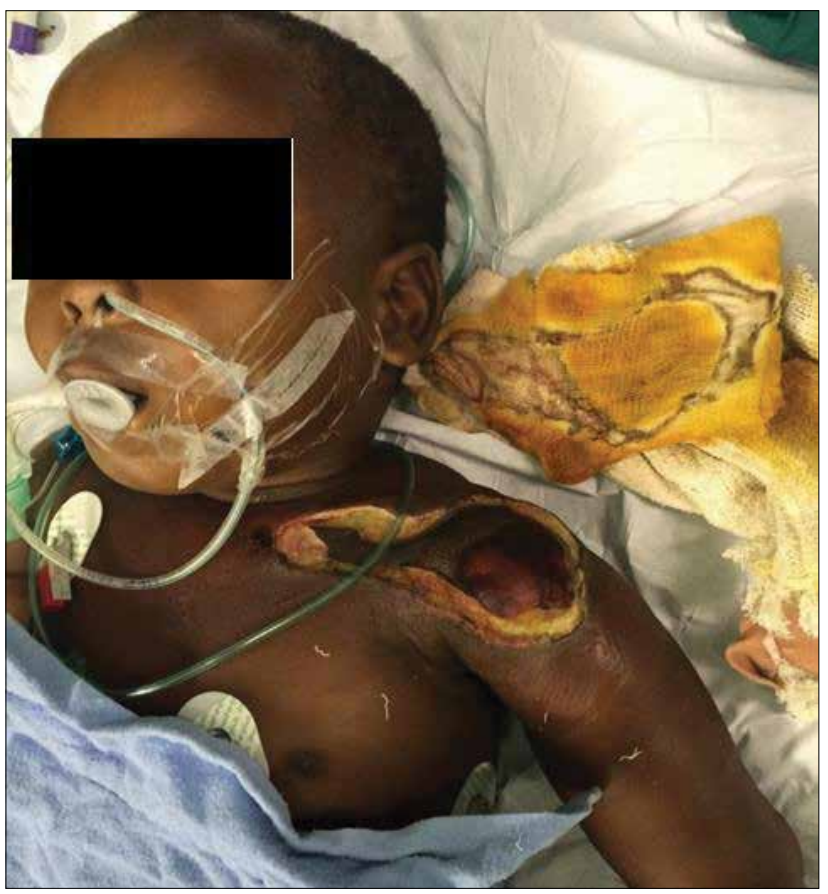

Fig. 8. Child with severe sepsis complicating snakebite to left shoulder.

soft-tissue necrosis is also unknown. In a retrospective study from Thailand, antivenom was found to have no effect on the development of dermatonecrosis after green pit viper envenomation. ${ }^{[25]}$ Current guidelines maintain that there is a role for antivenom administration for predominantly cytotoxic snakebite. ${ }^{[16,21]}$ Owing to the significant potential risks of antivenom administration, ${ }^{[4,16]}$ a risk/benefit evaluation needs to be employed in each individual case. ${ }^{[26]}$

We maintain that the most important aspect of managing these patients after the acute phase is good initial debridement and robust postoperative care.

\section{Study limitations}

This analysis was confined to snakebites with the most severe necrosis requiring formal debridement in theatre. Anaerobic culture was not performed, as methods of specimen collection and culture require protection from oxygen for anaerobic organism identification. This procedure is not routine and was not implemented for the purpose of the study. Quantitative bacterial cultures were not performed. Incomplete data from lost or inappropriately processed specimens may have compromised results.

\section{Conclusions and recommendations}

We recommend that antibiotics should be used in selected cases, treatment being commenced only when the results of sensitivity tests are known. In cases of severe sepsis where empirical antibiotic therapy is needed, use of ciprofloxacin is recommended in adults and children, as this covers Gram-negative Enterobacteriaceae and E. faecalis, avoids the need for combination therapy, and is considered safe in children. ${ }^{[27,28]}$ It is recommended that debridement be delayed until necrosis demarcates, usually after 5 - 7 days. Rare cases where the patient becomes systemically toxic should be treated with emergency debridement.

1. Blaylock R. Epidemiology of snakebite in Eshowe, KwaZulu-Natal, South Africa. Toxico 200 166. https://doi.org/10.1016/j.toxicon.2003.11.019

2. Coetzer PW, Tilbury CR. The epidemiology of snakebite in northern Natal. S Afr Med J 1982;62(7):206-217. 3. Wilkinson D. Retrospective analysis of snakebite at a rural hospital in Zululand. S Afr Med J 1994;84(12):844847.

4. Wood D, Webb C, DeMeyer J. Severe snakebites in northern KwaZulu-Natal: Treatment modalities and outcomes. S Afr Med J 2009;99(11):814-818.

Blaylock RS. Antibiotic use and infection in snakebite victims. S Afr Med J 1999;89(8):874-876.

6. Chen CM, Wu KG, Chen CJ, Wang CM. Bacterial infection in association with snakebite: A 10-year experience in a northern Taiwan medical center. J Microbiol Immunol Infect 2011;44(6):456-460. https:// doi.org/ $10.1016 /$ j.jmii.2011.04.0

Garg A, Sujatha S, Garg J, Acharya NS, Chandra Parija S. Wound infections secondary to snakebite. J Infect Developing Ctries 2009;3(3):221-223. https://doi:10.3855/jidc.39

8. Mao YC, Liu PY, Hung DZ, et al. Bacteriology of Najaatra snakebite wound and its implications for antibiotic therapy. Am J Trop Med Hyg 2016;94(5):1129-1135. https://doi.org/10.4269/ajtmh.15-0667

Ashe S, Chisale MGP. Guidelines for the Prevention and Clinical Management of Snakebite in Africa Brazzaville: World Health Organization, Regional Office for Africa, 2010.

10. Chippaux JP. Estimate of the burden of snakebites in sub-Saharan Africa: A meta-analytic approach Toxicon 2011;57(4):586-599. https://doi.org/10.1016/j.toxicon.2010.12.022

11. Kasturiratne A, Wickremasinghe AR, de Silva N, et al. The global burden of snakebite: A literature analysis and modelling based on regional estimates of envenoming and deaths. PLoS Med 2008;5(11):e218. https:// doi.org/10.1371/journal.pmed.0050218

12. Wood D, Sartorius B, Hift R. Estimating the burden of snakebite on public hospitals in KwaZulu Natal, South Africa. Wilderness Environ Med 2016;27(1):53-61. https:// doi.org/10.1016/j.wem.2015.11.005

3. Habib AG, Kuznik A, Hamza M, et al. Snakebite is under appreciated: Appraisal of burden from West Africa. PLoS Negl Trop Dis 2015;9(9):e0004088. https://doi.org/10.1371/journal.pntd.0004088

14. Habib AG. Public health aspects of snakebite care in West Africa: Perspectives from Nigeria. J Venom Anim Toxins Incl Trop Dis 2013;19:27. https://doi.org/10.1186/1678-9199-19-27

5. Spiller HA, Bosse GM. Prospective study of morbidity associated with snakebite envenomation. J Toxicol Clin Toxicol 2003;41(2):125-130. https://doi.org/10.1081/CLT-120019127

6. Blaylock R. Normal oral bacteria flora from some southern African snakes. Onderstepoort J Vet Res 2001;68(3):175.

17. Blaylock RS. The identification and syndromic management of snakebite in South Africa. S Afr Fam Pract 2014;47(9):48-53. https://doi.org/10.1080/20786204.2005.10873288

18. Blaylock RS. Antibacterial properties of KwaZulu Natal snake venoms. Toxicon 2000;38(11):1529-1534. https://doi.org/10.1016/S0041-0101(00)00085-4

19. Tilbury CR. Observations on the bite of the Mozambique spitting cobra (Naja mossambica mossambica).S Afr Med J 1982;61(9):308-313.

20. Vermaak SS, Visser A, le Roux TLB. A deadly bed partner: m’Fesi (Mozambique spitting cobra). SA Orthop J 2010;9(4):58-62.

21. Muller GJ, Modler H, Wium CA, Veale DJH, Marks CJ. Snake bite in southern Africa: Diagnosis and management. CME 2012;30(10):362-382.

22. Hall EL. Role of surgical intervention in the management of crotaline snake envenomation. Ann Emerg Med 2001;37(2):175-180. https://doi.org / $10.1067 /$ mem.2001.113373

23. Rojnuckarin P, Chanthawibun W, Noiphrom J, Pakmanee N, Intragumtornchai T. A randomized, doubleblind, placebo-controlled trial of antivenom for local effects of green pit viper bites. Trans R Soc Trop Med Hyg 2006;100(9):879-884. https://doi.org/10.1016/.t.trstmh.2005.10.006

24. Tanen DA, Danish DC, Clark RF. Crotalidae polyvalent immune Fab antivenom limits the decrease in panen DA, Danish DC, Clark RF. Crotalidae polyvalent immune Fab antivenom limits the decrease in
perfusion pressure of the anterior leg compartment in a porcine crotaline envenomation model. Ann Emerg perfusion pressure of the anterior leg compartment in a porcine cr
Med 2003;41(3):384-390. https://doi.org/10.1067/mem.2003.80

25. Chotenimitkhun R, Rojnuckarin P. Systemic antivenom and skin necrosis after green pit viper bites. Clinical toxicology 2008;46(2):122-125. https://doi.org/10.1080/15563650701266826

26. Hadley GP. Snakebites in children in Africa: A practical approach to management. Surgery in Africa Monthly Reviews, 2006. http://ptolemy.ca/members/archives/2006/Snake_Bites.htm (accessed 25 July 2016)

27. Grady R. Safety profile of quinolone antibiotics in the pediatric population. Pediatr Infect Dis 2003;22(12):1128-1132. https://doi.org/10.1097/01.inf.0000101994.25947.12

28. Schaad UB. Fluoroquinolone antibiotics in infants and children. Infect Dis Clin North Am 2005;19(3):617 628. https://doi.org/10.1016/j.idc.2005.05.005

Accepted 19 December 2016 